\title{
Nathalie Freidel, La conquête de l'intime. Public et privé dans la "Correspondance" de Madame de Sévigné
}

\section{Laura Rescia}

\section{(2) OpenEdition}

1 Journals

\section{Edizione digitale}

URL: http://journals.openedition.org/studifrancesi/5604

DOI: $10.4000 /$ studifrancesi. 5604

ISSN: 2421-5856

\section{Editore}

Rosenberg \& Sellier

\section{Edizione cartacea}

Data di pubblicazione: 1 septembre 2011

Paginazione: 401-402

ISSN: 0039-2944

\section{Notizia bibliografica digitale}

Laura Rescia, "Nathalie Freidel, La conquête de l'intime. Public et privé dans la "Correspondance" de Madame de Sévigné», Studi Francesi [Online], 164 (LV | II) | 2011, online dal 30 novembre 2015, consultato il 13 janvier 2021. URL: http://journals.openedition.org/studifrancesi/5604 ; DOI: https:// doi.org/10.4000/studifrancesi.5604

Questo documento è stato generato automaticamente il 13 janvier 2021.

\section{(c) 9 (i) $\Theta$}

Studi Francesi è distribuita con Licenza Creative Commons Attribuzione - Non commerciale - Non opere derivate 4.0 Internazionale. 


\title{
Nathalie Freidel, La conquête de l'intime. Public et privé dans la "Correspondance" de Madame de Sévigné
}

\author{
Laura Rescia
}

\section{NOTIZIA}

NATHALIE FREIDEL, La conquête de l'intime. Public et privé dans la "Correspondance" de Madame de Sévigné, Paris, Champion, 2009, 732 pp.

1 Questo saggio, ancorato alla prospettiva socio-letteraria ma attento all'analisi stilistica e linguistica, prende le mosse da una considerazione strettamente storico-sociale. Assumendo il Seicento come epoca della separazione tra spazio pubblico e privato, l'A. si domanda come questo fattore abbia influito sull'elaborazione oltre che sulla ricezione della Correspondance. L'ipotesi di fondo è che in questa raccolta sia identificabile l'emergenza di una nuova configurazione del genere epistolare, che troverebbe espressione nei contenuti e nelle forme innovative delle lettere sevignane, lontane dai modelli dei segretari e dei manuali di corrispondenza dell'epoca, e per alcuni aspetti leggibili alla luce della categoria dell'autofiction.

2 Solidamente riferito alle acquisizioni critiche e al corpus sevignano stabilito da Roger Duchêne, il volume non intende ripercorrere l'evoluzione tematica dell'epistolario, pur considerando la cronologia quando questo si rende necessario, e si tiene sempre a giusta distanza dalla tentazione psicologista. I risultati dell'analisi vengono articolati in quattro sezioni. La prima è incentrata sulla ricognizione della divisione pubblico/ privato nel XVII secolo: poggiando sugli studi innovatori di Alain Viala e Hélène Merlin, viene strutturata sulle dimensioni politica, religiosa, letteraria e infine epistolare. Nella sfera politica, con il progressivo affermarsi dello Stato moderno, emerge la 
rappresentazione di un'opinione pubblica di cui Mme de Sévigné appare cosciente, che si differenzia dallo spazio privato, luogo del segreto. La scissione è segnalata dall'utilizzo di linguaggi e codici diversi, da riferimenti ad uno spazio sociale individuato nell'espressione "dessous des cartes», dalla demarcazione tra persona e personaggio, e da quella distanza dalla scena pubblica che la nobildonna, epigona della filosofia degli Essais, sembra essere capace di mantenere. Anche in materia religiosa Mme de Sévigné sembra concepire una sua originale spiritualità, che si confronta e acquisisce tratti delle principali correnti dell'epoca, ma si mantiene dubbiosa nei confronti del giansenismo, e addirittura ironica verso il misticismo quietista, optando per una posizione rispettosa delle istanze del cuore pur senza offuscare quelle della ragione. Le conseguenze della scissione pubblico/privato in campo letterario sono, come noto e qui sottolineato, apprezzabili sia nel mutamento dei ruoli dell'autore e del pubblico, allargatosi ad includere le donne, sia nel progressivo imporsi della lettura silenziosa. In virtù di queste due ultime prerogative la letteratura si apre alla sfera dell'intimità: questo cambiamento si riverbera su molti generi, oltre a quello epistolare. Alla questione critica relativa ai potenziali lettori della Correspondance, l'A. risponde allineandosi all'opinione di Duchêne, che considerava soltanto incidentale la circolazione delle lettere sevignane fuori dalla sfera familiare. L'ultimo capitolo di questa prima parte giunge a un'osservazione minuta delle caratteristiche della lettera intima, che dovrebbe rispondere, secondo l'A., a tre criteri, tutti posseduti dall'epistolario della marchesa: provenire da un ambito privato e rendere conto di circostanze precise e personali; essere concepita come spazio espressivo di un mittente ben identificato; indirizzarsi a un destinatario unico, ben presente nel dialogo epistolare.

3 Nella seconda sezione si analizza l'epistolario attraverso la varietà degli ambienti che vi sono evocati, riconducibili a tre dicotomie: la corte / la città, Parigi / la provincia, il salone / la camera o giardino. Questi luoghi sono indagati sia come spazi descritti dalla marchesa, sia come luoghi della sua produzione epistolare. La disaffezione di Mme de Sévigné nei confronti della corte emergerebbe da una partecipazione distaccata agli eventi di uno spazio sociale che tuttavia è molto presente nella narrazione: l'A. riprende e avvalora la lettura di Duchêne a tal riguardo, sottolineando come le informazioni relative alla corte siano secondarie rispetto al piacere di raccontarla, e alla funzione emotiva che sempre emerge dalle lettere. La città viene descritta in una sequenza di tableaux, attraverso l'evocazione di un circuito familiare, sociale e amicale che ruota intorno al Marais, contenuto in luoghi circoscritti e tuttavia, contrariamente alla corte, aperti all'universo della provincia. Questa diviene nell'epistolario della marchesa un luogo familiare, non semplicemente un rifugio dove apprezzare le bellezze della natura, quanto piuttosto uno spazio fertile per la scrittura, utile a creare intimità con la corrispondente. Sévigné è lontana dunque dal deprezzare il decentramento dalla capitale, vissuto in tutta la sua ricchezza creativa. Se la sua retraite risulta non priva di una dimensione sociale, e corrisponde ad una vera e propria moda dell'epoca, per la marchesa il ritiro rivela, con il passare degli anni, un atteggiamento di abbandono della vanitas, una tensione alla ricerca del sé, che essa trasforma in art de vivre non dissimile dalla lezione salesiana. Anche gli interni descritti nella Correspondance rivelano una predilezione per quei cabinets che, nello spazio domestico secentesco, in movimento verso la distinzione degli ambienti pubblici e privati all'interno delle dimore, rimanda all'esigenza di interiorità. Tuttavia l'A. sottolinea come, anche in questo aspetto, le frontiere tra pubblico e privato rivelino una sicura porosità. 
4 La terza parte del volume, a nostro avviso meno essenziale, ripete in parte osservazioni già annotate nella seconda: viene studiato il rapporto individuo-società nella sfera mondana, nel circolo delle amicizie, nel rapporto con la famiglia. Sévigné, che pur dimostra di apprezzare l'individualità intesa come capacità di sfumare le necessità e gli obblighi sociali in comportamenti originali e non interamente conformi a tali imperativi, non disattende mai codici e vincoli sociali. Nel capitolo più interessante di questa sezione, intitolato «Nébuleuse du moi», si analizza l'articolazione del discorso su di sé in un'epoca, come il Grand Siècle, di assoluta reticenza a riguardo. La marchesa affronta questa tensione adottando le massime precauzioni retoriche, suggerite dalla necessità di politesse, corretta da una garbata ironia, attraverso la quale, tra nascondimenti e svelamenti, ella si rappresenta. Emerge così ancora la duplicità del suo atteggiamento, nel tentativo di ridurre le distanze, pur mantenendo quelle imposte dai codici sociali.

5 La quarta parte si concentra sullo spazio dell'intimità. Si analizza il concetto di segreto, inteso sia come codice linguistico che come luogo di occultamento dei sentimenti. Il problema dell'ineffabilità si verifica con la presenza di ellissi, citazioni implicite e allusioni, un insieme di strategie che consente di consolidare la connivenza tra autrice e destinataria principale delle lettere. L'espressione di emozioni e sentimenti è controllata, con un ritegno tutto consonante al gusto classicista, a cui si associa il timore di autocelebrazione esplicitato dalla marchesa. La cartografia del vocabolario degli affetti, e l'analisi puntuale delle tematiche dello spazio e del tempo, della presenza e dell'assenza, completano lo studio, dimostrando e avvalorando l'ipotesi complessiva di una poetica dell'incertezza e del movimento come cifra ideologica e stilistica fondamentale della Correspondance.

6 Conclude il volume un'articolata bibliografia, l'indice delle lettere citate e dei nomi. Merita menzione negativa il fatto che le edizioni Champion abbiano dimostrato un'esagerata disattenzione nei confronti dell'impaginazione di questo testo che, automatizzata e non verificata, ha prodotto innumerevoli errori di sillabazione, rendendone la lettura assai faticosa. 